\title{
Effect of Ester Side Chains on the Aggregation State and Surface Properties of Poly(dialkylfumarate)s
}

\author{
Sung Boo ChOI, ${ }^{\dagger}$ Atsushi TaKahara, Naoyuki AmaYA, ${ }^{*}$ \\ Yoshishige MURATA, ${ }^{*}$ and Tisato KaJIYAMA ${ }^{\dagger \dagger}$ \\ Department of Applied Chemistry, Faculty of Engineering, Kyushu University, \\ Hakozaki, Higashi-ku, Fukuoka 812, Japan \\ * Tsukuba Research Laboratory, Nippon Oil \& Fats Co., Ltd., Tokodai 5-10, \\ Toyosato-cho, Tsukuba, Ibaraki 300-26, Japan
}

(Received June 6, 1988)

\begin{abstract}
The aggregation state and surface properties of poly(dialkylfumarate)s (PDAFs) with various alkyl ester groups have been studied by X-ray diffraction, dynamic viscoelastic measurements, contact angle analysis, and X-ray photoelectron spectroscopy (XPS). PDAFs in the rigid rod-like molecular structure showed the mechanical $\alpha$-absorption behavior in a temperature range above $450 \mathrm{~K}$, which was attributed to the glassy-rubbery phase transition. The $\beta$-absorption behavior was apparently dependent on the bulkiness of the ester side chain. The intermolecular distance of rigid rod like molecules increased with the number of carbon atoms in the bulky ester group. The angular dependent XPS and contact angle measurements revealed that the polymers have low surface free energy due to the orientation of hydrophobic alkyl chains around the core composed of backbone chain.

KEY WORDS Poly(dialkylfumarate) / Rod-Like Molecule / $\alpha$-Absorption / $\beta$-Absorption / X-Ray Photoelectron Spectra / Surface Free Energy /
\end{abstract}

The thermal and mechanical properties of various glassy polymers have been investigated extensively. ${ }^{1}$ Most of these glassy vinyl polymers show a glass transition temperature lower than $400 \mathrm{~K}$ due to the flexibility of the main chain. However, the glassy heat-resistant polymers with high thermal stability have been investigated for the practical application. Poly(dialkylfumarate)s (PDAFs) prepared by the radical polymerization of fumaric acid derivatives have been evaluated as novel polymeric materials with excellent thermal stability. $^{2,3}$ Since the repeat unit of the polymers has two bulky ester groups in both sides of the main chain, PDAFs have characteristics of a rigid rod-like structure because of the high repulsive force between neighboring ester groups $^{4,5}$ Also, the polymers do not form crystalline phases and show characteristic properties of glassy polymers.

In this study, the effects of alkyl ester substituents on the aggregation state and surface preperties of PDAFs have been studied.

\section{EXPERIMENTAL}

\section{Materials}

The chemical structures and ester substituents of PDAFs used in this experiment are shown in Figure 1. In order to reveal the effects of ester substituents, two series of PDAFs were used in this study. One of the two ester substituents, $\mathrm{R}_{1}$, is isopropyl alcohol (iP) or tertbutyl alcohol (tB). Other substituents, $R_{2}$, are

\footnotetext{
${ }^{\dagger}$ Present address: Department of Chemical Engineering, Dongguk University, Seoul 100, Korea.

${ }^{++}$To whom correspondence should be addressed.
} 


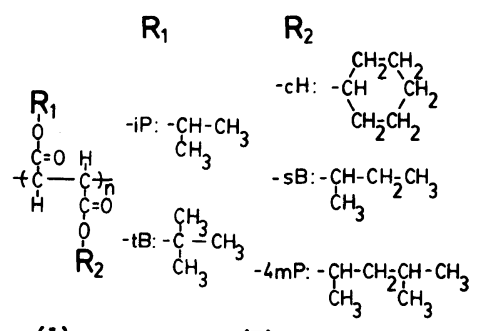

(1)

(II)

Figure 1. Chemical structures of poly(alkyl isopropylfumarate)s, poly(alkyl $t$-butylfumarate)s, and substituent ester groups.

Table I. Properties of poly(dialkylfumarate)s

\begin{tabular}{|c|c|c|c|c|}
\hline Polymer & $\mathrm{R}_{1}$ & $\mathbf{R}_{2}$ & $\bar{M}_{n}$ & $d / \mathrm{g} \mathrm{cm}^{-3}$ \\
\hline $\mathrm{P}(\mathrm{cH}-\mathrm{iPF})$ & $-\mathrm{cH}$ & $-i P$ & 77100 & 1.124 \\
\hline $\mathrm{P}(\mathrm{sB}-\mathrm{iPF})$ & $-s B$ & $-i P$ & 89000 & 1.094 \\
\hline $\mathrm{P}(4 \mathrm{mP}-\mathrm{iPF})$ & $-4 m P$ & $-i \mathrm{P}$ & 101300 & 1.078 \\
\hline $\mathrm{P}(\mathrm{iP}-\mathrm{tBF})$ & $-i P$ & $-\mathrm{tB}$ & 88500 & 1.084 \\
\hline $\mathrm{P}(\mathrm{cH}-\mathrm{tBF})$ & $-\mathrm{cH}$ & $-\mathrm{tB}$ & 49500 & 1.100 \\
\hline$P(s B-t B F)$ & $-s B$ & $-\mathrm{tB}$ & 63200 & 1.076 \\
\hline $\mathrm{P}(4 \mathrm{mP}-\mathrm{tBF})$ & $-4 \mathrm{mP}$ & $-\mathrm{tB}$ & 41800 & 1.038 \\
\hline
\end{tabular}

cyclohexyl alcohol (cH), sec-butyl alcohol (sB), and 4-methylpentyl alcohol (4mP). These polymers are designated as $\mathrm{P}(\mathrm{R}-\mathrm{iPF})$ or $\mathrm{P}(\mathrm{R}-$ tBF), where $R$ indicates various types of ester substituents. The film specimen of these polymers was prepared by casting a $1 \mathrm{wt} \%$ bezene solution on a clean glass plate at room temperature. This film was subsequently dried in vacuo for $12 \mathrm{~h}$. Table I summarizes the ester substituents, the number average molecular weight, and density of poly(alkyl isopropylfumarate)s (P(R-iPF)) and poly(alkyl $t$-butylfumarate)s (P(R-tBF)) used in this study.

\section{Characterization}

A wide angle X-ray diffraction (WAXD) and dynamic viscoelastic measurements were carried cut in order to characterize the aggregation state of PDAFs. The wide angle X-ray diffraction intensity curves were measured with a X-ray diffractometer (Rigaku Co., Ltd.) employing X-ray source of $\mathrm{Cu}-K_{\alpha}$. The temperature dependences of dynamic viscoelas-

ticity were measured with Rheovibron DDVIIC (Orientec Co., Ltd.) at $11 \mathrm{~Hz}$ under dried nitrogen flow.

The characterization of surface property was carried out by means of X-ray photoelectron spectroscopy (XPS) and contact angle measurements. Contact angles of the water and methylene iodide droplets on the materials surface were measured with a contact angle goniometer CA-D (Kyowa Kaimenkagaku Co., Ltd.) at 293 K. XPS spectra were obtained with a ESCA750 photoelectron spectrometer (Shimadzu Co., Ltd.) using $\mathrm{Mg}-K_{\alpha} \mathrm{X}$-ray source operated at $8 \mathrm{kV}$ and $30 \mathrm{~mA}$. The angular dependent XPS was measured by changing the angle between the sample suface and the direction of the analyzer from $90^{\circ}$ to $15^{\circ}$, in order to obtain the depth profile of the surface composition. A charging shift is referred to as the $C_{1 \mathrm{~s}}$ line emitted from a neutral carbon.

\section{RESULTS AND DISCUSSION}

\section{Molecular Aggregation State of Poly (dial- kylfumarate)s}

A wide angle $\mathrm{X}$-ray diffraction pattern was obtaind in order to ivestigate the aggregation state of PDAFs. It showed a strong Debye ring at the Bragg spacing of $1.1-1.4 \mathrm{~nm}$. For the quantitative analysis, the wide angle X-ray diffraction intensity curve was measured. The broad diffraction peak was observed at the Bragg spacing of 1.09 to $1.32 \mathrm{~nm}$. The magnitude of this Bragg spacing increased with the number of carbon atoms in the ester side chain. Yamada et al. observed a similar magnitude of Bragg spacing for poly(diisopropylfumarate) (PDiPF). ${ }^{4,5}$ Also, the differential radial distribution curve of PDiPF suggested that this Bragg spacing corresponds to the intermolecular distance of PDiPF., 4 Figure 2 shows the relationships between the magnitude of density and the intermolecular distance of poly(dialkylfumarate)s. The density of PDAFs is higher than conventional vinyl 


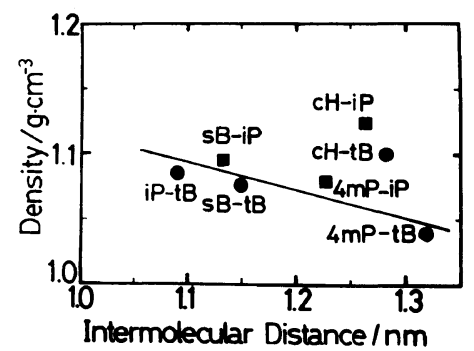

Figure 2. Relationships between density and intermolecular distance for poly(dialkylfumarate)s.

polymers. ${ }^{1}$ The intermolecular distance increased with the number of carbon atoms in ester substituents. On the other hand, the magnitude of density decreased with an increase in intermolecular distance. This suggests that the packing state of polymer chains depends on the shape and dimension of ester substituents and also, the core of PDAFs molecule is surrounded by hydrophobic substituents, resulting in a rod-like structure owing to repulsive force between ester substituent groups.

\section{Thermomechanical Behavior}

Dynamic viscoelastic measurements were carried out in order to reveal thermal molecular motions in PDAFs. Figure 3 shows the temperature dependences of dynamic storage modulus, $E^{\prime}$ and loss modulus, $E^{\prime \prime}$ for poly $(s-$ butyl isopropylfumarate) (P(sB-iPF)) and poly ( $s$-butyl $t$-butylfumarate) $(\mathrm{P}(\mathrm{sB}-\mathrm{tBF}))$. The temperature dependences of $E^{\prime}$ and $E^{\prime \prime}$ for the polymers showed remarkable difference owing to the different molecular aggregations in these side chain structures. The $\alpha$ absorption corresponding to the molecular motion of the main chain was observed at above $480 \mathrm{~K}^{4.5}$ and was confirmed from a steep decrease in $E^{\prime}$ at this temperature region. The $E^{\prime}$ for $\mathrm{P}(\mathrm{sB}$ iPF) decreased from $1 \mathrm{GPa}$ to $0.1 \mathrm{GPa}$ over the range of $\beta$ absorption temperature and was maintained at $0.1 \mathrm{GPa}$ in the $\beta-\alpha$ temperature range. On the other hand, $E^{\prime}$ for $\mathrm{P}(\mathrm{sB}-\mathrm{tBF}) \mathrm{did}$ not show remarkable decrease at the $\beta$-absorption region, and $\alpha$-absorption was ob-



Figure 3. Temperature dependence of dynamic storage modulus, $E^{\prime}$ and loss modulus, $E^{\prime \prime}$ for poly $(s$-butyl isopropylfumarate) and poly(s-butyl $t$-butylfumarate) at $11 \mathrm{~Hz}$.

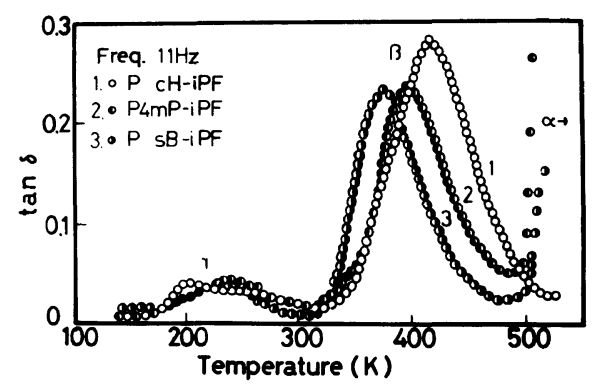

Figure 4. Temperature dependence of mechanical loss tangent, $\tan \delta$, for poly(alkyl isopropylfumarate)s with various alkyl chains.

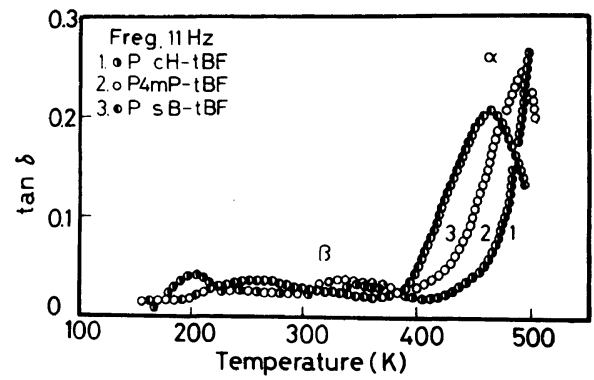

Figure 5. Temperature dependence of mechanical loss tangent, $\tan \delta$, for poly(alkyl $t$-butylfumarate)s with various alkyl chains.

served at around $450 \mathrm{~K}$ which is lower than that for $\mathrm{P}(\mathrm{sB}-\mathrm{iPF})$. These results indicate that the bulkiness of the side chain influences the rigidity of molecular chain below an $\alpha$-absorption temperature region. The differential scanning calorimetric curve showed the exothermic behavior above this temperature to be due to the decomposition of poly(dialkylfumarate). The decomposition temperature for 
Table II. Surface free energy for poly(dialkylfumarate)s

\begin{tabular}{|c|c|c|c|c|c|}
\hline \multirow{2}{*}{ Surface } & \multicolumn{2}{|c|}{ Contact angle $\left({ }^{\circ}\right)$} & \multicolumn{3}{|c|}{ Component energy/ $\mathrm{erg} \mathrm{cm}^{-2}$} \\
\hline & $\mathrm{H}_{2} \mathrm{O}$ & $\mathrm{CH}_{2} \mathrm{I}_{2}$ & $\gamma_{\mathrm{sv}}^{\mathbf{d}}$ & $\gamma_{\mathrm{sv}}^{\mathrm{h}}$ & $\gamma_{\mathrm{sv}}$ \\
\hline $\mathrm{P}(\mathrm{cH}-\mathrm{iPF})$ & 90.5 & 51.8 & 31.5 & 1.9 & 33.4 \\
\hline $\mathrm{P}(\mathrm{sB}-\mathrm{iPF})$ & 94.2 & 57.0 & 28.9 & 1.5 & 30.4 \\
\hline $\mathrm{P}(4 \mathrm{mP}-\mathrm{iPF})$ & 115.0 & 65.0 & 27.2 & 0.2 & 27.4 \\
\hline $\mathrm{P}(\mathrm{iP}-\mathrm{tBF})$ & 99.0 & 59.4 & 28.2 & 0.7 & 27.9 \\
\hline $\mathrm{P}(\mathrm{cH}-\mathrm{tBF})$ & 94.9 & 56.2 & 29.5 & 1.2 & 30.7 \\
\hline $\mathrm{P}(\mathrm{sB}-\mathrm{tBF})$ & 96.7 & 56.6 & 29.6 & 0.9 & 30.5 \\
\hline $\mathrm{P}(4 \mathrm{mP}-\mathrm{tBF})$ & 121.0 & 70.0 & 24.7 & 0.6 & 25.3 \\
\hline
\end{tabular}

poly(alkyl isopropylfumarate) was higher than that for poly(alkyl $t$-butylfumarate). This is ascribed to the thermal stability of the side chain. In the case of $t$-butyl group, isobutane was produced due to thermal decomposition at ca. $450 \mathrm{~K}$.

Figures 4 and 5 show the temperature dependences of $\tan \delta$ for poly(alkyl isopropylfumarate)s and poly(alkyl-t-butyl fumarate)s. The $\beta$-absorption corresponding to the molecular motion of the side chain was observed at around $400 \mathrm{~K}$ for poly(alkyl isopropylfumarate). The $\beta$-absorption temperature increased with an increase in the bulky size of the ester substituents. Yamada et al. reported that a large increase in Bragg spacing at the $\beta$ absorption region accompanies a slight decrease in $E^{\prime} .^{5,6}$ This slight decrease in $E^{\prime}$ was also observed for the present series of poly(alkyl isopropylfumarate)s. The $\beta$-absorption process for poly(alkyl $t$-butylfumarate) was observed at $300 \mathrm{~K}$ which is lower than that observed for poly(alkyl isopropylfumarate)s. Also, the relaxation strength was smaller than that observed for poly(alkyl isopropylfumarate). This indicates that only a small part of the side chain begins its molecular motion at this temperature.

\section{Surface Characteristics of Poly(dialkylfuma- rate)s}

The surface free energy for poly(alkyl isopropylfumarate)s and poly(alkyl $t$-butylfuma- rate)s was evaluated from the contact angles of water and methylene iodide on the solid surface at $293 \mathrm{~K}$. Surface free energy was calculated by the procedure of Owens and Wendt. ${ }^{6}$ The contact angles of water and methylene iodide, the total surface free energy, $\gamma_{\mathrm{sv}}$, its dispersion component, $\gamma_{\mathrm{sv}}^{\mathrm{d}}$ and hydrogen-bonding component, $\gamma_{\mathrm{sv}}^{\mathrm{h}}$ for each solid surfaces are summarized in Table II. The general characteristic of a PDAF surface is hydrophobic. The magnitudes of surface free energy and its component for poly(cyclohexyl isopropylfumarate) are almost same as that of polyethylene. ${ }^{6}$ This indicates that the surface of poly(dialkylfumarate) is completely covered with hydrocarbon groups. Since this molecule forms a rigid rod-like structure, the hydrophobic alkyl achains surrounds its backbone. The magnitude of the total surface free energy decreased with an increase in the number of methylene groups in the alkyl ester substituents. The hydrogen-bonding component is very small compared with the dispersion one. The magnitude of the hydrogen-bonding component decreased with an increase in the number of carbon atoms in the ester substituents. Since a methyl group has lower surface free energy than that of a methylene group, the experimental results of surface free energy indicate the orientation of hydrophobic alkyl groups around the backbone chain. The surface of poly(alkyl $t$-butylfumarate)s showed smaller surface free energy compared with that 

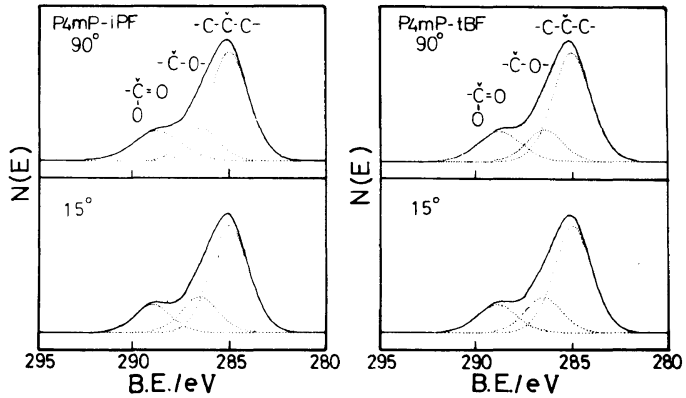

Figure 6. Carbon 1s spectra for poly(4-methylpentyl isopropylfumarate) and poly(4-methylpentyl $t$-butylfumarate) at the emission angle of photoelectron of $15^{\circ}$ and $90^{\circ}$

Table III. Analytical results of XPS for poly(dialkylfumarate)s

\begin{tabular}{|c|c|c|c|}
\hline \multirow{2}{*}{ Polymer } & \multirow{2}{*}{ Bulk $(\mathrm{O} / \mathrm{C})$} & \multicolumn{2}{|c|}{$\operatorname{XPS}\left(\mathrm{O}_{\mathrm{ls}} / \mathrm{C}_{\mathrm{ls}}\right)$} \\
\hline & & $90^{\circ}$ & $15^{\circ}$ \\
\hline $\mathrm{P}(\mathrm{cH}-\mathrm{iPF})$ & 0.308 & 0.342 & 0.277 \\
\hline $\mathrm{P}(\mathrm{cH}-\mathrm{tBF})$ & 0.285 & 0.326 & 0.265 \\
\hline $\mathrm{P}(4 \mathrm{mP}-\mathrm{PPF})$ & 0.308 & 0.318 & 0.284 \\
\hline $\mathrm{P}(4 \mathrm{mP}-\mathrm{tBF})$ & 0.285 & 0.333 & 0.298 \\
\hline$P(s B-i P F)$ & 0.364 & 0.374 & 0.361 \\
\hline $\mathrm{P}(\mathrm{sB}-\mathrm{tBF})$ & 0.333 & 0.298 & 0.284 \\
\hline$P(\mathrm{iP}-\mathrm{tBF})$ & 0.364 & 0.405 & 0.359 \\
\hline
\end{tabular}

of poly(alkyl isopropylfumarate)s in the case that the substituent of $R_{1}$ is same. This is ascribed to the bulkiness of the $t$-butyl group.

Surface chemical analysis of PDAFs was carried out by angular dependent X-ray photoelectron spectra. Figure 6 shows the $C_{1 \mathrm{~s}}$ spectra for poly $(4 \mathrm{mP}-\mathrm{iPF})$ and poly $(4 \mathrm{mP}-\mathrm{tBF})$ at the emission angles of photoelectron of $15^{\circ}$ and $90^{\circ}$. The analytical depth decreased with a decrease in emission angle. ${ }^{7}$ A peak at $285.0 \mathrm{eV}$ was assigned to the neutral carbon of alkyl substituent and backbone $\mathrm{C}-\mathrm{C}$ chains $\left(-\mathrm{C}-\mathrm{C}^{*}-\mathrm{C}-\right)$. The peak observed at $268.5 \mathrm{eV}$ was assigned to the ethereal carbon $\left(-\mathrm{C}^{*}-\mathrm{O}-\right.$ CO-). Also, the $\mathrm{C}_{1 \mathrm{~s}}$ peak at $288.8 \mathrm{eV}$ corresponds to the carbonyl carbon. Table III summarizes the results of XPS analysis. The calculated number ratios of oxygen to carbon atoms in bulk $(\mathrm{O} / \mathrm{C})$ were estimated from chemical structures of monomers. The ratio of oxygen to carbon atoms $(\mathrm{O} / \mathrm{C})$ decreased with a decrease in the emission angle. This indicates that the concentration of alkyl carbon increased with a decrease in analytical depth. The high concentration of alkyl group may be closely related to the low surface free energy of PDAFs.

\section{CONCLUSION}

The surface and bulk characterization of PDAFs with various ether substituents were carried out by X-ray diffraction, dynamic viscoelastic measurements, X-ray photoelectron spectroscopy and contact angle measurements. The onset of $\beta$-absorption for poly(alkyl $t$ butylfumarate) was located at lower temperature than that for poly(alkyl isopropylfumarate). However, the relaxation strength of $\beta$-absorption for poly(alkyl $t$-butylfumarate) was smaller than that for poly(alkyl isopropylfumarate) due to rigidity of the backbone chain. The wide angle X-ray diffraction measurements suggest the existence of aggregates of rigid rod-like molecules in solid state. The XPS and contact angle measurements revealed that the surface of poly(dialkylfumarate) has small free energy due to the orientation of hydrophobic alkyl groups around the backbone chain.

\section{REFERENCES}

1. F. A. Bovey and F. H. Winslow (ed), "Macromolecules-An Introduction to Polymer Science," Academic Press, New York, N.Y., 1979.

2. T. Otsu and N. Toyoda, J. Macromol. Sci., Chem., A19, 1011 (1983).

3. T. Otsu, H. Minami, N. Toyoda, and T. Yasuhara, Makromol. Chem. Suppl., 21, 133 (1985).

4. K. Yamada, M. Takayanagi, and Y. Murata, Rep. Prog. Polym. Phys., Jpn., 27, 321 (1984).

5. K. Yamada, M. Takayanagi, and Y. Murata, Polymer, 27, 1054 (1986).

6. D. K. Owens and R. C. Wendt, J. Appl. Polym. Sci., 21, 1741 (1969).

7. T. Kajiyama, N. Morotomi, S. Hiraoka, and A. Takahara, Chem. Lett., 1737 (1987). 